\title{
Truncating variant burden in high-functioning autism and pleiotropic effects of LRP1 across psychiatric phenotypes
}

\author{
Bàrbara Torrico, PhD; Alex D. Shaw, PhD; Roberto Mosca, PhD; Norma Vivó-Luque, BSc; \\ Amaia Hervás, MD; Noèlia Fernàndez-Castillo, PhD; Patrick Aloy, PhD; \\ Mònica Bayés, PhD; Janice M. Fullerton, PhD; Bru Cormand, PhD*; Claudio Toma, PhD*
}

\begin{abstract}
Background: Previous research has implicated de novo and inherited truncating mutations in autism-spectrum disorder. We aim to investigate whether the load of inherited truncating mutations contributes similarly to high-functioning autism, and to characterize genes that harbour de novo variants in high-functioning autism. Methods: We performed whole-exome sequencing in 20 high-functioning autism families (average $I Q=100$ ). Results: We observed no difference in the number of transmitted versus nontransmitted truncating alleles for high-functioning autism (117 v. 130, $p=0.78$ ). Transmitted truncating and de novo variants in high-functioning autism were not enriched in gene ontology (GO) or Kyoto Encyclopedia of Genes and Genomes (KEGG) categories, or in autism-related gene sets. However, in a patient with high-functioning autism we identified a de novo variant in a canonical splice site of LRP1, a postsynaptic density gene that is a target for fragile $X$ mental retardation protein (FRMP). This de novo variant leads to in-frame skipping of exon 29 , removing 2 of 6 blades of the $\beta$-propeller domain 4 of $L R P 1$, with putative functional consequences. Large data sets implicate $L R P 1$ across a number of psychiatric disorders: de novo variants are associated with autism-spectrum disorder $(p=0.039)$ and schizophrenia $(p=$ 0.008) from combined sequencing projects; common variants using genome-wide association study data sets from the Psychiatric Genomics Consortium show gene-based association in schizophrenia $\left(p=6.6 \times \mathrm{E}^{-07}\right)$ and in a meta-analysis across 7 psychiatric disorders $\left(p=2.3 \times \mathrm{E}^{-03}\right)$; and the burden of ultra-rare pathogenic variants has been shown to be higher in autism-spectrum disorder $(p=1.2 \times$ $\mathrm{E}^{-05}$ ), using whole-exome sequencing from 6135 patients with schizophrenia, 1778 patients with autism-spectrum disorder and 7875 controls. Limitations: We had a limited sample of patients with high-functioning autism, related to difficulty in recruiting probands with high cognitive performance and no family history of psychiatric disorders. Conclusion: Previous studies and ours suggest an effect of truncating mutations restricted to severe autism-spectrum disorder phenotypes that are associated with intellectual disability. We provide evidence for pleiotropic effects of common and rare variants in the LRP1 gene across psychiatric phenotypes.
\end{abstract}

\section{Introduction}

Autism-spectrum disorder (ASD) is characterized by impairments in social interactions and communication, and by repetitive behaviours. Appearing in approximately $1 \%$ of the general population, ASD is one of the most prevalent disorders in childhood. ${ }^{1}$ The clinical phenotype is heterogeneous and includes a broad range of comorbidities, such as epilepsy, language impairment, anxiety, sleep disorders or attention-deficit/hyperactivity disorder. ${ }^{2}$ However, one of the most remarkable clinical features in ASD is intellectual disability, which is present in a considerable proportion of patients ${ }^{3}$ and associated with the most severe phenotypic outcomes across the spectrum. ${ }^{4}$ Patients with ASD who have a higher intelligence quotient (IQ > 70) and average or high cognitive abilities are often considered to be part of a more homogeneous clinical group referred to as highfunctioning autism (HFA).

Recent family studies confirm that genetic factors play a considerable role in ASD. Heritability $\left(h^{2}=0.8\right)$ is one of the highest among neuropsychiatric disorders, but specific genetic risk factors remain largely unknown, and only a small proportion of the approximately 1000 genes estimated to be involved in ASD have been identified. ${ }^{5}$

Correspondence to: B. Cormand, Departament de Genètica, Microbiologia i Estadística, Facultat de Biologia, Universitat de Barcelona, 08028 Barcelona, Spain, bcormand@ub.edu; C. Toma, Neuroscience Research Australia, Margarete Ainsworth Building, Barker Street, Randwick NSW 2031, Sydney, Australia, c.toma@ neura.edu.au

*These authors contributed equally to this work.

Submitted Oct. 10, 2018; Revised Jan. 21, 2019; Revised Jan. 26, 2019; Accepted Feb. 8, 2019; Published online on May 16

DOI: 10.1503/jpn.180184 
Genetic studies conducted over the last 2 decades converge on a genetic model in which ASD genetic liability is shaped by both multiple common variants of small effect size and a discrete number of rare variants with higher penetrance. The first genome-wide association studies (GWAS) pointed at several common variants in $\mathrm{ASD}_{,}^{6-8}$ which were not replicated in a recent well-powered study of European populations. ${ }^{9}$ Although common risk variants are estimated to explain from about $20 \%$ to $50 \%$ of the genetics of $\mathrm{ASD}^{10}{ }^{10}$ larger cohorts are needed to identify individual allelic contributions. ${ }^{9}$ As well, recent efforts combining several large international GWAS data sets in a meta-analysis failed to identify genome-wide significant hits. ${ }^{11}$

Larger sample sizes and increased sensitivity in wholeexome sequencing (WES) or whole-genome sequencing studies have exponentially increased the identification of novel risk genes, starting to resolve most of the missing heritability in psychiatric disorders. Several sequencing studies have implicated both rare de novo variants and rare inherited single nucleotide variations in ASD. ${ }^{12-14}$ The first WES studies implicated de novo point mutations in disease pathogenesis in singleton ASD families, ${ }^{15-18}$ estimating that this mutation class may explain between $5 \%$ and $20 \%$ of genetic liability. ${ }^{16,19,20}$ De novo variants are considered highly deleterious, and an excess of de novo truncating gene variants has been found in probands with ASD. ${ }^{17}$ Interestingly, several independent studies have found a correlation between the higher burden of de novo truncating variants and lower IQ in ASD. ${ }^{15,16,18}$ Our group was the first to assess the impact of inherited rare variants in multiplex ASD families, ${ }^{21}$ suggesting that apart from de novo variants, inherited truncating variants also play a significant role in pathogenesis, with a higher burden in patients with ASD and a clear effect on probands with intellectual disability. ${ }^{21}$ The contribution of inherited truncating variants in ASD was replicated later in a large sample by comparing probands and their unaffected siblings. ${ }^{22}$

However, it is still unclear whether inherited truncating mutation burden is etiologically relevant in all patients with ASD or if it is prominent only in severe cases associated with intellectual disability. In this study we aimed to: (1) determine whether rare inherited truncating mutations are distinctly implicated in HFA; (2) identify molecular pathways or biological categories in ASD by considering the entire pool of severe mutations, including de novo and inherited truncating variants; and (3) identify potential novel candidate genes for ASD.

\section{Methods}

\section{Selection of participants and phenotypic assessment}

From our collection of ASD families, ${ }^{23,24}$ we selected 20 singleton Spanish families without any other psychiatric history among relatives. All probands had HFA, defined as a fullscale IQ greater than 70 (IQ mean $\pm \mathrm{SD}=100 \pm 14.7$; range $=$ 80-135). Clinical descriptions are provided in Appendix 1, Supplement and Table S1, available at jpn.ca/180184-a1.
Exome sequencing and WES-based genetic relatedness analysis

The sequenced sample included 60 participants (40 parents and 20 probands with ASD). We performed exome enrichment on $3 \mu \mathrm{g}$ of genomic DNA extracted from blood, and the exome libraries were applied to an Illumina flowcell for paired-end sequencing on a HiSeq2000 instrument (Illumina) using 76 base reads. Detailed bioinformatic analysis is provided in Appendix 1, Supplement. On average, individuals had $82.1 \%$ of the target covered by $>10$ reads (Appendix 1, Table S2). We confirmed familial relationships by genomewide identity-by-descendent analysis in PLINK, ${ }^{25}$ using WES-derived genotypes (Appendix 1, Supplement).

\section{Variant selection: rare truncating alleles and de novo variants}

We defined rare variants as those having a minor allele frequency $<1 \%$ in dbSNP135. The truncating alleles selected included nonsense, indels leading to frame-shift, variants in canonical splice sites and start-lost changes. For each family, we considered truncating alleles, both transmitted to the proband with ASD and nontransmitted (i.e., only in parents). Statistical analyses are described in detail in Appendix 1. We also examined de novo variants. We validated rare truncating alleles and de novo variants $(n=263)$ by Sanger sequencing. During variant validation, we also included 9 unaffected siblings from 8 families to assess the transmission of inherited truncating alleles from parents.

\section{Enrichment analyses}

We tested the enrichment of genes carrying inherited truncating alleles and de novo variants against all gene ontology (GO) categories and Kyoto Encyclopedia of Genes and Genomes (KEGG) pathways. We also performed enrichment analyses using the same pool of genes against several gene sets potentially related to ASD: namely, genes encoding postynaptic density proteins (PSD), ${ }^{26}$ fragile-X mental retardation protein (FMRP) targets ${ }^{27}$ and de novo variants previously found in autism and schizophrenia. ${ }^{28}$ Both analyses were performed by: (1) matching genes carrying potentially truncating or de novo variants to genes randomly drawn from the genome, after approximate matching by exome-enriched coding-sequence length and genic constraint missense Z-score (http:/ / exac.broadinstitute.org), ${ }^{29}$ and (2) calculating an empirical $p$ value for observed data in each functional category, using a null distribution of overlap counts from 1000 randomly drawn gene sets, as described previously. ${ }^{30}$

\section{Effect of LRP1 de novo mutations on splicing}

We performed functional predictions for the de novo splice-site variant in LRP1 (chr12:57573110A/G) using 3 tools: MaxEntScan (http://genes.mit.edu/burgelab/maxent/ Xmaxentscan_scoreseq.html), SPANR (http://tools.genes .toronto.edu) and Human Splicing Finder (http:/ / www.umd .be/HSF3). To assess functional consequences at the RNA level, we used complementary DNA (cDNA) from the peripheral blood mononuclear cells of SJD_33.3 (a patient with ASD; 
Appendix 1, Supplement). We separated polymerase chain reaction (PCR) products from primers designed in exons 28 and 30 using 10\% polyacrylamide gel electrophoresis, and we measured the intensity of the 2 resulting bands using a semiquantitative method (Appendix 1, Supplement).

\section{Protein modelling of LRP1: consequence of in-frame skipping} of exon 29

We modelled the LRP1 wild-type protein using the SWISSMODEL platform, ${ }^{31}$ which was enquired to search homologous templates using the LRP1 full length sequence (Appendix 1). The selected model was generated from template 3s94.1.A, corresponding to the structure of the $\beta$-propeller domains 1 and 2 of the human LRP6 gene (PDB ID 3s94). ${ }^{32}$ We examined the LRP1 protein model that includes exon 29 using both SWISSMODEL and the Robetta server. ${ }^{33}$

\section{LRP1 gene network analysis}

We investigated whether LRP1 was included in a network of genes previously implicated in ASD using Ingenuity Pathway Analysis software (www.ingenuity.com; Appendix 1). We computed the most likely network of interactions given a pool of 75 genes with a high probability of involvement in ASD selected from the SFARI database (categories $S$ and 1; https://gene.sfari.org/database/gene-scoring/).

\section{Effect of the LRP1 mutated form on inflammatory biomarkers}

We extracted RNA from 2 different lymphoblastoid cell lines, 1 from patient SJD_33.3 and another from a control, and we obtained their cDNA (Appendix 1). We performed quantitative real-time PCR and enzyme-linked immunoassays for the following cytokines: interleukin-6 (IL-6) and tumour necrosis factor $\alpha$ (TNF $\alpha$; proinflammatory response); and IL-10 (anti-inflammatory response). Details of these experiments and primers are provided in Appendix 1, Supplement and Table S3.

\section{LRP1: de novo, common and rare variant analyses in psychiatric disorders}

We used 2 databases to identify previous de novo variants in LRP1. ${ }^{34,35}$ We used NPdenovo to assess the overall de novo association between $L R P 1$ and several neuropsychiatric conditions: ASD (6118 families), schizophrenia (1164 families), epilepsy (647 families) and intellectual disability (1101 families) ${ }^{34}$

We calculated gene-level association for common variants and meta-analysis in LRP1 with MAGMA ${ }^{36}$ (Appendix 1), using data sets for people of European descent only, derived from summary statistics of the Psychiatric Genomics Consortium GWAS (https://med.unc.edu/ pgc/results-and-downloads). ${ }^{11,37-42}$

We investigated the LRP1 single nucleotide polymorphisms (SNPs) significantly associated with schizophrenia in the last GWAS (rs12814239 and rs12826178) ${ }^{39}$ for their effect on LRP1 expression using Stanley Medical Research Institute Neuropathology Consortium and BrainCloud data (Appendix 1, Supplement). We also assessed expression of LRP1 across different developmental stages in human brain using 3 data sources: the Genotype-Tissue Expression project (https:/ / gtexportal.org/home), the Human Brain Transcriptome (http:/ /hbatlas.org) and BrainCloud (https:// getbraincloud.com).

We performed analysis of rare variants in LRP1 using the sequencing data of schizophrenia, ASD and control cohorts (Appendix 1, Supplement). The selection of potentially etiologic variants is described in Appendix 1, Supplement. We first performed a burden analysis using RVTESTS, ${ }^{43}$ only in data sets containing both cases and controls from the same sequencing platform and project (Swedish schizophrenia case-control and Baylor College of Medicine autism casecontrol data sets). We then used a $\chi^{2}$ statistic to separately compare the schizophrenia patient sample (6135 cases) and combined ASD data sets (1778 cases) with the combined control data sets (7875 individuals).

\section{Results}

\section{Inherited truncating alleles in HFA}

A total of 247 truncating alleles were validated after WES in 20 HFA families (47\% indel-frameshift, 37\% nonsense, 16\% canonical splice-site variants) and are listed in Appendix 1, Table S4. We assessed whether the number of truncating alleles transmitted to HFA probands was higher than nontransmitted alleles, and found no significant difference across all families (average of proportions $=47 \%, z=0.283, p=0.78$; Table 1), or when considering each family individually

\begin{tabular}{|c|c|c|c|c|c|}
\hline & Total $^{*}$ & Transmitted & Nontransmitted & MeanProp $†$ & $p$ value \\
\hline \multicolumn{6}{|l|}{ Truncating alleles } \\
\hline Probands $(n=20)$ & 247 & 117 & 130 & $47 \%$ & 0.78 \\
\hline Unaffected siblings $(n=9)$ & 111 & 46 & 65 & $42 \%$ & 0.64 \\
\hline \multicolumn{6}{|c|}{ Truncating alleles (brain-expressed) } \\
\hline Probands $(n=20)$ & 144 & 71 & 73 & $49 \%$ & 0.93 \\
\hline Unaffected siblings $(n=9)$ & 59 & 25 & 34 & $43 \%$ & 0.66 \\
\hline
\end{tabular}


(Appendix 1, Table S5). Moreover, we observed no difference after restricting the analysis to truncating alleles in brain-expressed genes (average of proportions $=49 \%, z=$ $0.077, p=0.93$; Table 1 ). We observed a similar ratio of transmitted/nontransmitted alleles in unaffected siblings (average of proportions $=42 \%, z=0.47, p=0.64$; Table 1 and Appendix 1, Table S6).

\section{Identification of de novo variants in HFA}

Next, we examined the impact of de novo variants across the 20 HFA families and validated 16 variants: 13 missense, 2 synonymous and 1 splicing variant (Appendix 1, Table S7). Missense variants, predicted pathogenic by both SIFT and PolyPhen-2, were found in PCDH15, ADD3, GALNT6 and TEX14. A potential functional de novo variant was found in a canonical acceptor splice site in the LRP1 gene in 1 proband (Appendix 1, Figure S1).

\section{Enrichment analysis of inherited truncating and de novo variants}

We performed enrichment analysis for GO categories and KEGG pathways of the combined pool of highly damaging variants, including both inherited truncating alleles and de novo variants found in the 20 probands with HFA. Although we found categories with a plausible role in ASD in the top hits (brain morphogenesis, $p=0.001$; neurotransmitter receptor complex, $p=0.003$; histidine metabolism, $p=0.023$ ), none was significant after multiple correction (Appendix 1, Table S8 and Table S9).

Enrichment analysis against gene sets that have been previously implicated in psychiatric disorders ${ }^{28,30}$ found no evidence of enrichment (Table 2). However, LRP1 was the only mutation-carrying gene in our study that was present in all gene sets considered - namely, the PSD genes, de novo variants in ASD, de novo variants in schizophrenia and FMRP target genes (Table 2) — warranting further investigation of this candidate gene.

\section{LRP1 de novo splice-site variant leads to skipping of exon 29 with functional consequences}

The LRP1 de novo variant found in proband SJD_33.3 (chr12:57573110, A/G) is located in a highly conserved canonical splice site and is absent from the gnomAD database (http://gnomad.broadinstitute.org/). The AG to GG change was predicted to be functional and highly deleterious, according to 3 splicing-based analysis tools: MaxEntScan (score: 4.12), SPANR (dPSI-Wild-type: 0; dPSI-Mutant: -12.95) and HSF (wild-type score: 86 ; mutant score: 57 ), predicting a disrupted acceptor site of exon 29 (Fig. 1A and Appendix 1, Figure S1). Examination of $L R P 1 \mathrm{mRNA}$ from the patient's blood lymphocytes confirmed an in-frame skipping of exon 29 (Fig. 1B). The expression levels of transcripts from the mutated and wild-type alleles were similar (Appendix 1). The LRP1 de novo variant was also present in the patient's buccal cells from a saliva sample, indicating a germline mutational origin and the presence of the abnormal transcript in all tissues where LRP1 is expressed, including brain. Moreover, this de novo mutation was absent in 2 unaffected siblings from the same family.

To assess the potential functional consequences of exon 29 skipping (p.1580-1655del) at the protein level, we performed a modelling study of the LRP1 protein domains (NP_002323.2). Exon 29 encodes part of a YWTD $\beta$-propeller domain of LRP1 (Fig. 1C). Each of the $\beta$-propeller domains is the result of 6 YWTD repeats that are organized in 6 4 -stranded $\beta$-sheets (blades) arranged radially about a central symmetry axis. We generated a structural model of the third and fourth LRP1 $\beta$-propeller domains, based on the homology of LRP6, which showed that exon 29 encodes the first 2 blades of the fourth $\beta$-propeller domain (Fig. 1D). When we modelled the mutated form of LRP1 lacking the exon 29, the sequence alignment matched the $\beta$-propeller domains 3 and 5 , whereas the $\beta$-propeller domain 4 segment was unaligned and could not be folded into a globular structure. However, the poor quality of the model does not exclude the possibility that the mutated $\beta$-propeller domain 4 may fold into an ordered structure.

To investigate whether LRP1 is involved in specific ASD networks of protein-protein interactions, we used Ingenuity Pathway Analysis software with 75 genes that have previously been implicated in ASD. Interestingly, genes strongly associated with ASD such as SHANK3, FMR1, SYNGAP1 and GRIN2B were found in the same network with $L R P 1$, downstream of $L R P 1$ in the signalling pathway (Appendix 1, Figure S2).

Given findings that suggest the direct involvement of LRP1 in inflammatory response, ${ }^{44-46}$ we assessed whether the mutated form of LRP1 may compromise inflammatory response by measuring IL-6, TNF $\alpha$ and IL-10. We found lower mRNA

Table 2: Gene-set enrichment analysis of transmitted truncating alleles and de novo variants in 20 high-functioning autism probands (130 genes)

\begin{tabular}{|c|c|c|c|}
\hline Gene set ( $n$ genes) & $\begin{array}{l}\text { Observed } \\
\text { (expected) }\end{array}$ & $\begin{array}{l}\text { Empirical } \\
p \text { value }\end{array}$ & Genes \\
\hline Genes expressed in the postsynaptic density $(1435)^{26}$ & $6(9.9)$ & 0.95 & TBC1D24, UGP2, ADD3, CDH2, GRIN2B, LRP1* \\
\hline Fragile $\mathrm{X}$ mental retardation protein target genes $(835)^{27}$ & $4(6.4)$ & 0.91 & GRIN2B, NPAS2, DIDO1, LRP1* \\
\hline De novo variants found in autism $(768)^{28}$ & $8(7.2)$ & 0.43 & $\begin{array}{c}\text { METTL16, GRIN2B, EFCAB5, CACNA1S, MUC17, UTP20, } \\
\text { LRP1, " DNAH11 }\end{array}$ \\
\hline De novo variants found in schizophrenia $(694)^{29}$ & $9(7.1)$ & 0.25 & $\begin{array}{c}\text { ZNF551, FILIP1, IGSF22, CLTCL1, MYH7B, CACNA1S, } \\
\text { MUC17, VPS13C, LRP1* }\end{array}$ \\
\hline
\end{tabular}

*The recurrent gene $L R P 1$.

The same analysis was performed in nontransmitted truncating alleles (128 genes; not significant). 
levels for all 3 cytokines in the patient's lymphoblastoid cell line compared with a control (Fig. 2). We obtained comparable results at the protein level for IL-10 and TNF $\alpha$ (Appendix 1, Fig. S3), but IL-6 was not detected in the assay. When we treated the lymphoblastoid cell line with lipopolysaccharide to trigger an inflammatory response, we observed a physiologic proinflammatory response of IL-6 in the control, but not in the patient cell line (Fig. 2).

LRP1 is ubiquitously expressed, and found in all brain tissues, especially the cerebellum (Appendix 1, Figure S4A). Expression reported in the BrainCloud and Human Brain Transcriptome databases showed higher or increasing expression of the gene during fetal or postnatal development, and relatively stable expression over the rest of development in all brain tissues (Appendix 1, Figure S4B-C).
Involvement of LRP1 in psychiatric disorders by comprehensive analysis of large data sets

Genome-wide significant associations in schizophrenia have recently been reported for SNPs in the LRP1 region: rs324017 $\left(p=2.12 \times \mathrm{E}^{-08}\right.$, including replication), $\operatorname{rs12814239}(p=1.48 \times$ $\left.\mathrm{E}^{-09}\right)$ and $\mathrm{rs12826178}\left(p=2.02 \times \mathrm{E}^{-12}\right.$, including replication; Appendix 1, Figure S5). ${ }^{39}$ Two of these, rs12814239 (p. C1261C), a synonymous variant in LRP1, and rs12826178 (intergenic) are in linkage disequilibrium $\left(\mathrm{D}^{\prime}=0.95 ; r^{2}=0.74\right.$; Europeans in 1000 genomes; Appendix 1, Fig. S5). We used BrainCloud and Stanley Medical Research Institute Neuropathology Consortium data sets to assess the potential effects of schizophrenia risk alleles on the expression of $L R P 1$, but rs12814239 and rs12826178 were not directly genotyped in

A

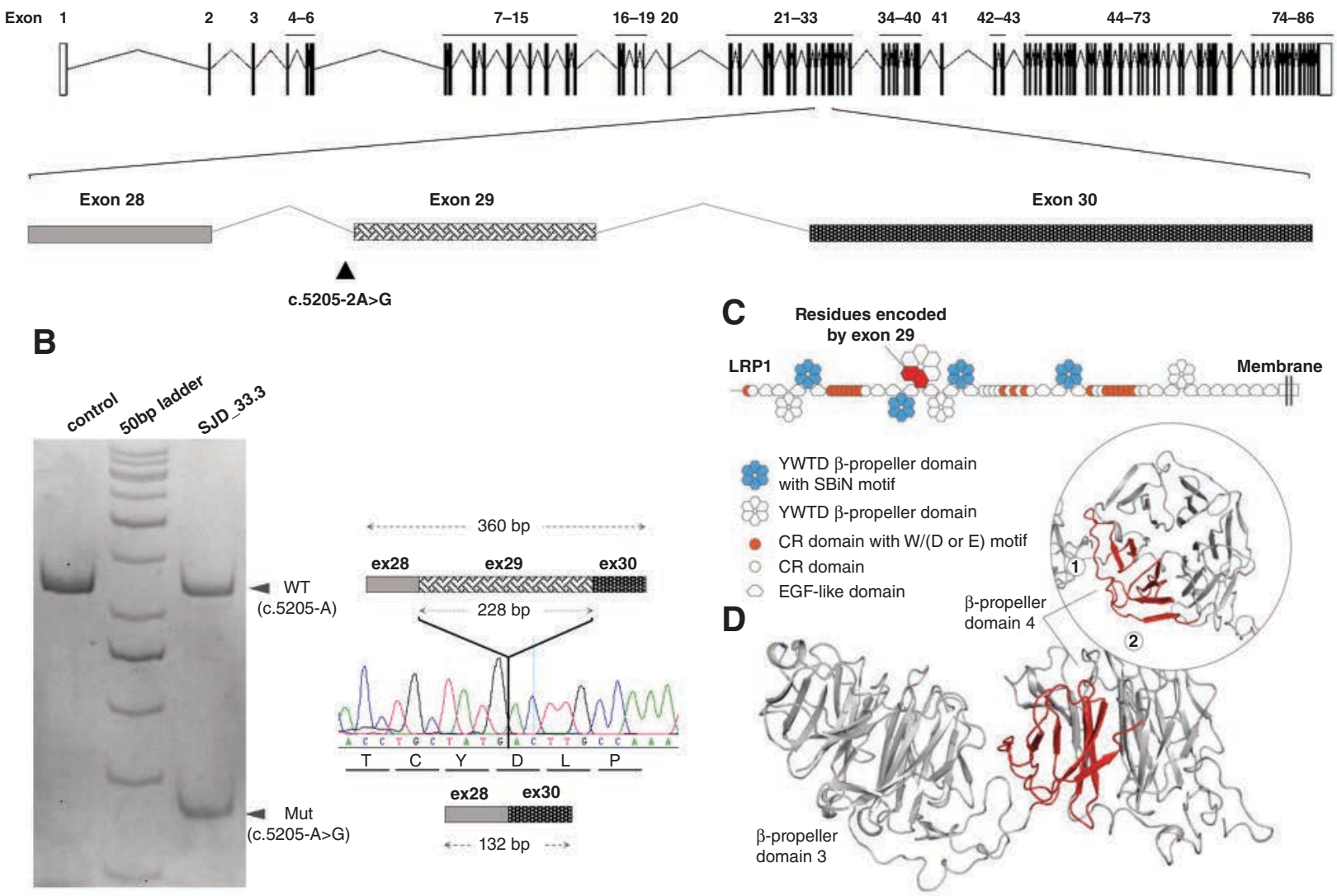

Fig. 1: Effect of the identified de novo change on LRP1 splicing. (A) Schematic structure of the LRP1 gene (NM_002332), with exons 28 to 30 amplified below. The mutation site is indicated by a triangle (c.5205-2A $>G$ ). (B) Polymerase chain reaction (PCR) analysis of $L R P 1$ complementary DNA from lymphocytes visualized on polyacrylamide gel. For the wild-type (WT) transcript, the PCR amplicon of 360 bp included a fragment of exon 28, the entire exon 29 and a fragment of exon 30, whereas the mutated transcript (Mut) generated a smaller fragment (132 bp) lacking exon 29 (76 amino acids), which generates an in-frame transcript. The 2 transcripts spanning exon 28 to 30 are represented by the sequenced band of the mutated allele. (C) Schematic representation of $L R P 1$ domains with the $\beta$-propellers as hexagons. The region encoded by exon 29 in $\beta$-propeller 4 is in red. (D) Cartoon model for the LRP1 domain $\beta$-propellers 3 and 4 obtained from the template of the $\beta$-propeller domains 1 and 2 of LRP6 (PDB ID 3s94). The skipping of exon 29 led to the removal of the first 2 blades of 6 from $\beta$-propeller 4. 
these data sets, nor were other SNPs that would have served as reasonable surrogates $\left(r^{2}>0.7\right)$.

The identification of a functional de novo variant in the LRP1 gene in an ASD family and the reported associations across the LRP1 locus in schizophrenia prompted us to explore the impact of common, rare and de novo variants of this gene in several large psychiatric data sets.

The NPdenovo and denovo-db databases report de novo variants in LRP1 in projects on psychiatric disorders (Appendix 1, Table S10). These de novo variants include 3 highly pathogenic variants, all absent from the gnomAD database: a stop mutation from a patient with schizophrenia (p.Y2200*), a frame-shift from a patient with intellectual disability (p. Q3380Sfs*72) and an exon 29 variant in a patient with ASD, which was likely to disrupt an exonic splicing enhancer site with an effect potentially similar to that reported in patient SJD_33.3. The NPdenovo data showed association between the de novo variant in LRP1 with ASD $(p=0.039)$, intellectual disability $(p=0.008)$ and schizophrenia $(p=0.008)$.

We also explored the possible contribution of common variants in LRP1 by performing a gene-based association study using summary statistics from the Psychiatric Genomics Consortium GWAS data in European populations, which suggest that common variants in LRP1 increase risk of schizophrenia (gene-based $p=6.6 \times \mathrm{E}^{-07}$ ), and a trend for attention-deficit/hyperactivity disorder and bipolar disorder, but not in the ASD sample (Table 3). A meta-analysis combining data from 7 psychiatric disorders implicates LRP1 common variants across these conditions $\left(p=2.3 \times \mathrm{E}^{-03}\right.$; Table 3).

We performed a burden analysis using a combined multivariate and collapsing method to assess the impact of predicted pathogenic rare or ultra-rare variants (minor allele frequency $<0.0001$ ) of LRP1 in schizophrenia (Swedish casecontrol) and autism (Baylor College of Medicine case-control) data sets. We found no differences in schizophrenia $(p=0.63)$, but observed a significant burden in autism probands ( $p=$ 0.048). When data for each phenotype were combined (7875 controls, 6135 patients with schizophrenia and 1778 probands with ASD), we observed no difference in the ultra-rare variants between patients with schizophrenia and controls $(p=$ 0.52 ), but we did observe a higher burden in patients with $\operatorname{ASD}\left(p=1.2 \times \mathrm{E}^{-05}\right.$; Table 4 and Appendix 1, Table S11). These ultra-rare variants were not normally distributed along the domains and repeats of LRP1 in ASD or SCZ $\left(p<1 \times \mathrm{E}^{-05}\right)$, and interestingly, they appeared to cluster in the $\beta$-propeller

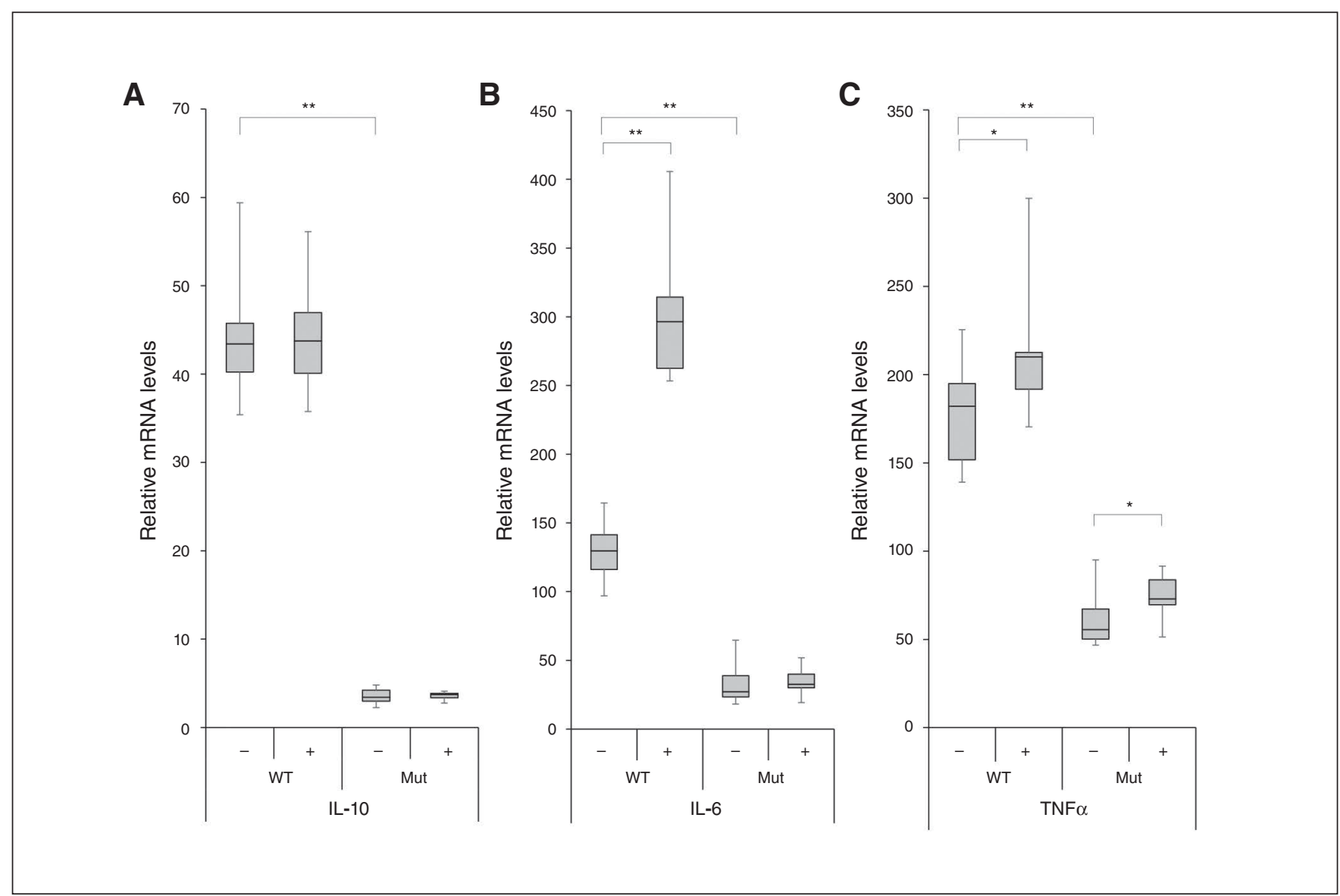

Fig. 2: The boxplots show the cytokine expression in immortalized lymphocyte cell lines from the patient (Mut) and a control (wild-type; WT) with $(+)$ or without $(-)$ treatment of lipopolysaccharide $(6 \mathrm{~h}$ at $1 \mathrm{mg} / \mathrm{mL})$. The mRNA quantifications of (A) interleukin-10 (IL-10), (B) interleukin-6 (IL-6) and (C) tumour necrosis factor $\alpha\left(\right.$ TNF $\alpha$ ) were normalized using ACTB as an endogenous reference. ${ }^{*} p<0.05 ;{ }^{* \star} p<0.0005$. 
domain 5 in ASD (Grubbs' test 1-tail $\alpha=0.05$; Appendix 1, Table S12).

\section{Discussion}

Recent sequencing studies have strongly implicated de novo and inherited pathogenic rare variants in ASD. ${ }^{12-22}$ However, these variants seem to have a varying impact across the heterogeneous ASD phenotype. While the contribution of de novo truncating variants has been extensively described in probands with ASD who have a lower IQ, insufficient data are available for inherited truncating variants. A previous study from our group found a higher burden for coinherited truncating variants in ASD sibling pairs, ${ }^{21}$ and the burden of these disruptive alleles correlated negatively with nonverbal IQ. ${ }^{21}$ Another study showed a significant impact for inherited gene-disrupting variants in probands with ASD and lowerthan-average IQ $(<100)$ compared with unaffected siblings. ${ }^{22}$ In the present study, we have assessed the impact of rare inherited truncating variants by comparing transmitted versus nontransmitted gene-truncating variants in singleton families with HFA to establish whether highly disruptive variants have a role in all cases of ASD, regardless their comorbidity with intellectual disability. Our results showed no preferential burden of transmission from parents to probands with HFA, suggesting that truncating alleles may not have a major role across the entire spectrum, but may be restricted to patients with ASD and intellectual disability.

Correlation between severe disrupting mutations and severe phenotypes has also been reported for other psychiatric diseases: a higher rate of rare truncating variants was found in patients with schizophrenia and intellectual disability, ${ }^{47}$ and in bipolar disorder the burden of inherited truncating alleles was negatively correlated with early age of onset. ${ }^{30}$ Ultra-rare disruptive alleles have also been associated with lower educational attainment in the general population ${ }^{48}$ suggesting that this class of genetic variants may negatively modulate cognitive function. In summary, all of these findings suggest a correlation between the burden of severe mutations and the severity of the disease phenotype.

In our study, we also performed enrichment analyses in the list of genes bearing transmitted truncating alleles and de novo variants in probands with HFA. We observed no enrichments when we considered this pool of genes, but interestingly, LRP1 appeared recurrently in all psychiatricrelated gene sets. The de novo variant in $L R P 1$ found in a patient with HFA is not in the gnomAD database (138632 sequenced individuals) and is predicted to disrupt an acceptor splice site. The consequence of this mutation at the RNA level is an in-frame skipping of exon 29, which removes 2 out of 6 radial blades of the $\beta$-propeller 4 of $L R P 1$. It is likely that the $\beta$-propeller 4 is not folded in a functional canonical domain, potentially compromising interactions at this and adjacent $\beta$-propellers 3 and 5 .

We then investigated the possible involvement of this gene in several psychiatric disorders by exploring a number of genetic data sets. We identified an association of de novo variants in LRP1 with autism and schizophrenia, an association of common variants at the gene level in schizophrenia and an association in a meta-analysis of 7 psychiatric disorders, with significant impact for ultra-rare pathogenic variants in ASD. These results implicate common and rare variants in the LRP1 gene across several psychiatric phenotypes. Interestingly, although $L R P 1$ is ubiquitously expressed, higher expression is found in the postnatal cerebral stages, when abnormalities in brain are observed in patients with ASD. ${ }^{49}$ Studies in mice have also suggested a peak of LRP1 expression during early postnatal brain development in several populations of cells, including radial glia, immature and mature neurons, microglia and astrocytes. ${ }^{50}$

LRP1 has a dual role, involved in endocytosis and signal transduction, and it binds approximately 40 extracellular ligands that mediate a multitude of physiologic processes. ${ }^{51}$ The de novo mutation found in a proband with HFA in this study affects the structure of a $\beta$-propeller domain, which may impair ligand dissociation and the formation of early endosomes; ${ }^{52}$ the high burden of damaging ultra-rare variants found in autism may abolish interactions with some of the numerous ligands; the common variants found associated with schizophrenia may exert their role in gene regulation, acting on the antisense RNA LRP1-AS transcribed from the $L R P 1$ locus that negatively regulates LRP1 expression, ${ }^{53}$ or regulating alternative transcripts such as the recently identified truncated spliced form of LRP1 (smLRP1). ${ }^{54}$

Considering the high number of ligands and pathways mediated by $L R P 1$, it is difficult to pinpoint a specific

\begin{tabular}{|c|c|c|c|c|c|}
\hline Condition & Cases/controls & SNPs tested & $p$ value & Top SNP & Top SNP $p$ value \\
\hline Attention-deficit/hyperactivity disorder & $19099 / 34194$ & 112 & 0.10 & rs34577247 & 0.002 \\
\hline Anorexia nervosa & $3495 / 10982$ & 149 & 0.41 & rs2228187 & 0.008 \\
\hline Autism-spectrum disorder & $6197 / 7377$ & 80 & 0.99 & rs11172113 & 0.25 \\
\hline Bipolar disorder & $20352 / 31358$ & 219 & 0.06 & rs10467125 & 0.001 \\
\hline Major depressive disorder & $59851 / 113154$ & 254 & 0.49 & rs113379328 & 0.006 \\
\hline Obsessive-compulsive disorder & $2688 / 7037$ & 137 & 0.36 & rs7398375 & 0.003 \\
\hline Schizophrenia & $33640 / 43456$ & 227 & $6.6 \times \mathrm{E}^{-07}$ & rs12814239 & $2.91 \times E^{-09}$ \\
\hline Meta-analysis & & & $2.3 \times E^{-03}$ & & \\
\hline
\end{tabular}

PCG2 = Psychiatric Genomics Consortium 2; SNP = single nucleotide polymorphism. 
Table 4: LRP1 burden analysis of ultra-rare variants in autismspectrum disorder and schizophrenia*

\begin{tabular}{lccc}
\hline Group & Individuals & $\begin{array}{c}\text { Pathogenic } \\
\text { ultra-rare } \\
\text { variants }\end{array}$ & $p$ value \\
\hline Controls & 7875 & 64 & - \\
Schizophrenia† & 6135 & 44 & 0.52 \\
Autism-spectrum disorder $\ddagger$ & 1778 & 35 & $1.2 \times \mathrm{E}^{-05}$ \\
\hline
\end{tabular}

*Total individuals used across different sequencing data sets and number of ultra-rare variants predicted to be pathogenic. The selection of variants includes missense, truncating variants and splice-site variants (full list of ultra-rare variants in Appendix 1,

Table S11, available at jpn.ca).

tWhole-exon sequencing from the Sweden Schizophrenia Population-Based CaseControl data set (dbGAP accession: phs000473.v2.p2).

†ARRA Autism Sequencing Collaboration (dbGAP accession: phs000298.v3.p2).

compromised pathophysiological process. However, several plausible hypotheses can be formulated in relation to its impact on postsynaptic complexes, and its role in inflammatory response, insulin signalling and lipid homeostasis. First, LRP1 encodes a PSD protein and plays a role in synaptic integrity and function at the postsynapses by regulating GRIA1, ${ }^{55}$ implicated in learning disabilities and autism through de novo gain-of-function mutations that cause constitutive calcium-channel opening. ${ }^{14}$ We found LRP1 in the same network of well-established ASD genes such as SHANK3, GRIN2B and SYNGAP1. Second, LRP1 may exert effects via a compromised inflammatory response. It regulates inflammation through c-Jun N-terminal kinase (JNK) and nuclear factor $\kappa \mathrm{B}(\mathrm{NF}-\mathrm{\kappa B})$ pathways and has a neuroprotective role in microglia. ${ }^{44-46}$ After inflammatory insult, the lymphoblastoid cell line of the patient carrying the functional LRP1 splice variant was not responsive in expressing the IL-6 cytokine, suggesting that this pathway may be compromised. ${ }^{56}$ Third, LRP1 may mediate insulin signalling in brain, forming a complex with the insulin receptor $\beta$ and regulating insulin signalling and glucose homeostasis in brain. ${ }^{57}$ Both processes are involved in synaptic plasticity, memory and learning. Interestingly, a stop mutation in the $X$-linked brain-expressed insulin substrate receptor 4 gene (IRS4) segregated in schizoaffective patients in an extended family. ${ }^{30}$ Finally, LRP1 may exert its effects via impairment of lipid homeostasis. Knockout mice showed that neuronal LRP1 is critical for cholesterol and lipid metabolism, and its defect leads to dendritic spine degeneration, synapse loss and neuroinflammation. ${ }^{58}$ However, it is likely that etiologic genetic variants of $L R P 1$ exert their role in psychiatric diseases by concurrently impairing more than one pathway at a time.

It is noteworthy that other lipoprotein receptors from the same family, such as LRP2 and LRP8, have previously been implicated in autism and psychosis, ${ }^{59,60}$ suggesting an emerging role for this gene family in psychiatric disorders.

\section{Limitations}

The WES was performed in a small number of patients with HFA; the small sample was related to the difficulty in recruiting patients with high cognitive performance and no family history of psychiatric disorders.
Moreover, although we considered the impact of common and rare variants in LRP1 in several psychiatric disorders, we did not examine other classes of potential risk variants, such as structural variants. Furthermore, we were not able to control for the potential effect of sample overlap among the Psychiatric Genomics Consortium data sets of the 7 psychiatric disorders used for the gene-based association test, which may represent a confounding issue in regards to estimations of pleiotropy. Finally, we were not able to find potential biological consequences for the schizophrenia-associated SNPs in LRP1.

\section{Conclusion}

Considering our previous and current results, and additional sequencing findings presented here, we provide evidence of a relationship between severe mutations and severe ASD phenotype: the accumulation of inherited truncating mutations leads to a more severe phenotype in autism, whereas their impact in patients with HFA appears limited. Furthermore, we show through a comprehensive analysis of de novo, common and rare variants that $L R P 1$ is a candidate gene with pleiotropic effects across multiple psychiatric phenotypes.

Acknowledgements: The authors are grateful to all patients and their families for their participation, and thank all clinical collaborators who contributed to the diagnosis of probands. C. Toma was supported by the European Union (Marie Curie, PIEF-GA-2009-254930) and B. Torrico by AGAUR (Generalitat de Catalunya). Financial support was received from Fundació La Marató de TV3 (092010), Fundación Alicia Koplowitz, AGAUR (2017SGR738), the Spanish Ministerio de Economía y Competitividad (SAF2015-68341-R) and the Australian National Medical and Health Research Council (NHMRC) Project Grant 1063960 and 1066177, and Program Grant 1037196. The research leading to these results also received funding from the European Union H2020 Program (H2020/2014-2020) under grant agreements 667302 (CoCA) and 643051 (MiND). P. Aloy acknowledges the support of the Spanish Ministerio de Economía y Competitividad (BIO2013-48222-R; BIO2016-77038-R) and the European Research Council (SysPharmAD: 614944). J.M. Fullerton was supported by the Janette Mary O'Neil Research Fellowship. N. Fernàndez-Castillo was supported by the Centro de Investigación Biomédica en Red de Enfermedades Raras (CIBERER). Exome sequencing services were provided by the National Centre for Genomic Analysis (CNAG). The authors also thank the CIBERER Biobank (www.ciberer-biobank.es/), Rafael Valdés-Mas and Xose S. Puente from the University of Oviedo (Spain) for support in bioinformatics analyses, and Barbara Toson (NeuRA) for support in statistical analyses.

Affiliations: From the Departament de Genètica, Microbiologia i Estadística, Universitat de Barcelona, Spain (Torrico, Vivó-Luque, Fernàndez-Castillo, Cormand, Toma); the Institute of Biomedicine, University of Barcelona, Barcelona, Spain (Torrico, Fernàndez-Castillo, Cormand, Toma); the Centro de Investigación Biomédica en Red de Enfermedades Raras (CIBERER), Barcelona, Spain (Torrico, Fernàndez-Castillo, Cormand, Toma); the Institut de Recerca Hospital Sant Joan de Déu, Esplugues de Llobregat, Barcelona, Spain (Torrico, Fernàndez-Castillo, Cormand); the Neuroscience Research Australia, Sydney, NSW, Australia (Shaw, Fullerton, Toma); the School of Medical Sciences, University of New South Wales, Sydney, NSW, Australia (Shaw, Fullerton, Toma); the Institute for Research in Biomedicine (IRB Barcelona) and the Barcelona Institute of Science and Technology, Barcelona, Spain (Mosca, Aloy); the Child and Adolescent Mental 
Health Unit, Hospital Universitari Mútua de Terrassa, Spain (Hervás); the Institució Catalana de Recerca i Estudis Avançats (ICREA), Barcelona, Spain (Aloy); and the Centro Nacional de Análisis Genómico (CNAG), Barcelona, Spain (Bayés).

Competing interests: None declared.

Contributors: B. Torrico, M. Bayés, B. Cormand and C. Toma designed the study. B. Torrico and $\mathrm{C}$. Toma acquired and analyzed the data, which A. Shaw, R. Mosca, N. Vivó-Luque, A. Hervás, N. Fernàndez-Castillo, P. Aloy, J. Fullerton and B. Cormand also analyzed. C. Toma wrote the article, which all authors reviewed. All authors approved the final version to be published and can certify that no other individuals not listed as authors have made substantial contributions to the paper.

\section{References}

1. Elsabbagh M, Divan G, Koh YJ, et al. Global prevalence of autism and other pervasive developmental disorders. Autism Res 2012;5:160-79.

2. Freitag CM. The genetics of autistic disorders and its clinical relevance: a review of the literature. Mol Psychiatry 2007;12:2-22.

3. Idring $\mathrm{S}$, Lundberg $\mathrm{M}$, Sturm $\mathrm{H}$, et al. Changes in prevalence of autism spectrum disorders in 2001-2011: findings from the Stockholm youth cohort. J Autism Dev Disord 2015;45:1766-73.

4. Vivanti G, Barbaro J, Hudry K, et al. Intellectual development in autism spectrum disorders: new insights from longitudinal studies. Front Hum Neurosci 2013;7:354.

5. Betancur C. Etiological heterogeneity in autism spectrum disorders: more than 100 genetic and genomic disorders and still counting. Brain Res 2011;1380:42-77.

6. Anney R, Klei L, Pinto D, et al. A genome-wide scan for common alleles affecting risk for autism. Hum Mol Genet 2010;19:4072-82.

7. Weiss LA, Arking DE. Gene Discovery Project of Johns H, et al. A genome-wide linkage and association scan reveals novel loci for autism. Nature 2009;461:802-8.

8. Wang $\mathrm{K}$, Zhang $\mathrm{H}$, Ma D, et al. Common genetic variants on 5 p14.1 associate with autism spectrum disorders. Nature 2009; 459:528-33.

9. Torrico B, Chiocchetti AG, Bacchelli E, et al. Lack of replication of previous autism spectrum disorder GWAS hits in European populations. Autism Res 2017;10:202-11.

10. Cross-Disorder Group of the Psychiatric Genomics C, Lee SH Ripke S, et al. Genetic relationship between five psychiatric disorders estimated from genome-wide SNPs. Nat Genet 2013;45:984-94.

11. Autism Spectrum Disorders Working Group of The Psychiatric Genomics C. Meta-analysis of GWAS of over 16,000 individuals with autism spectrum disorder highlights a novel locus at 10q24.32 and a significant overlap with schizophrenia. Mol Autism 2017;8:21.

12. Yuen RK, Thiruvahindrapuram B, Merico D, et al. Whole-genome sequencing of quartet families with autism spectrum disorder. Nat Med 2015;21:185-91.

13. RK CY, Merico D, Bookman M, et al. Whole genome sequencing resource identifies 18 new candidate genes for autism spectrum disorder. Nat Neurosci 2017;20:602-11.

14. Geisheker MR, Heymann G, Wang T, et al. Hotspots of missense mutation identify neurodevelopmental disorder genes and functional domains. Nat Neurosci 2017;20:1043-51.

15. O'Roak BJ, Vives L, Girirajan S, et al. Sporadic autism exomes reveal a highly interconnected protein network of de novo mutations. Nature 2012;485:246-50.

16. Iossifov I, O'Roak BJ, Sanders SJ, et al. The contribution of de novo coding mutations to autism spectrum disorder. Nature 2014;515: 216-21.
17. De Rubeis S, He X, Goldberg AP, et al. Synaptic, transcriptional and chromatin genes disrupted in autism. Nature 2014;515:209-15.

18. Kosmicki JA, Samocha KE, Howrigan DP, et al. Refining the role of de novo protein-truncating variants in neurodevelopmental disorders by using population reference samples. Nat Genet 2017;49:504-10.

19. Neale BM, Kou Y, Liu L, et al. Patterns and rates of exonic de novo mutations in autism spectrum disorders. Nature 2012;485:242-5.

20. Sanders SJ, He X, Willsey AJ, et al. Insights into autism spectrum disorder genomic architecture and biology from 71 risk loci. Neuron 2015;87:1215-33.

21. Toma C, Torrico B, Hervas A, et al. Exome sequencing in multiplex autism families suggests a major role for heterozygous truncating mutations. Mol Psychiatry 2014;19:784-90.

22. Krumm N, Turner TN, Baker C, et al. Excess of rare, inherited truncating mutations in autism. Nat Genet 2015;47:582-8.

23. Toma C, Torrico B, Hervas A, et al. Common and rare variants of microRNA genes in autism spectrum disorders. World J Biol Psychiatry 2015;16:376-86.

24. Torrico B, Fernandez-Castillo N, Hervas A, et al. Contribution of common and rare variants of the PTCHD1 gene to autism spectrum disorders and intellectual disability. Eur J Hum Genet 2015;23:1694-701.

25. Purcell S, Neale B, Todd-Brown K, et al. PLINK: a tool set for whole-genome association and population-based linkage analyses. Am J Hum Genet 2007;81:559-75.

26. Bayes A, van de Lagemaat LN, Collins MO, et al. Characterization of the proteome, diseases and evolution of the human postsynaptic density. Nat Neurosci 2011;14:19-21.

27. Darnell JC, Van Driesche SJ, Zhang C, et al. FMRP stalls ribosomal translocation on mRNAs linked to synaptic function and autism. Cell 2011;146:247-61.

28. Fromer M, Pocklington AJ, Kavanagh DH, et al. De novo mutations in schizophrenia implicate synaptic networks. Nature 2014;506:179-84

29. Lek M, Karczewski KJ, Minikel EV, et al. Analysis of protein-coding genetic variation in 60,706 humans. Nature 2016;536:285-91.

30. Toma C, Shaw D, Allcock R, et al. An examination of multiple classes of rare variants in extended families with bipolar disorder. Transl Psychiatry 2018;8:65.

31. Biasini M, Bienert S, Waterhouse A, et al. SWISS-MODEL: modelling protein tertiary and quaternary structure using evolutionary information. Nucleic Acids Res 2014;42:W252-8.

32. Cheng Z, Biechele T, Wei Z, et al. Crystal structures of the extracellular domain of LRP6 and its complex with DKK1. Nat Struct Mol Biol 2011;18:1204-10.

33. Song Y, DiMaio F, Wang RY, et al. High-resolution comparative modeling with RosettaCM. Structure 2013;21:1735-42.

34. Li J, Cai T, Jiang Y, et al. Genes with de novo mutations are shared by four neuropsychiatric disorders discovered from NPdenovo database. Mol Psychiatry 2016;21:298.

35. Turner TN, Yi Q, Krumm N, et al. denovo-db: a compendium of human de novo variants. Nucleic Acids Res 2017;45:D804-11.

36. de Leeuw CA, Mooij JM, Heskes T, et al. MAGMA: generalized gene-set analysis of GWAS data. PLOS Comput Biol 2015;11:e1004219.

37. Demontis D, Walters RK, Martin J, et al. Discovery of the first genome-wide significant risk loci for ADHD. Nat Genet 2019;51:63-75.

38. Wray NR, Ripke S, Mattheisen M, et al. Genome-wide association analyses identify 44 risk variants and refine the genetic architecture of major depression. Nat Genet 2018;50:668-81.

39. Schizophrenia Working Group of the Psychiatric Genomics C. Biological insights from 108 schizophrenia-associated genetic loci. Nature 2014;511:421-7.

40. Stahl E, Forstner A, McQuillin A, et al. Genomewide association study identifies 30 loci associated with bipolar disorder. Nat Genet 2019;51:793-803. 
41. Duncan L, Yilmaz Z, Gaspar H, et al. Significant locus and metabolic genetic correlations revealed in genome-wide association study of anorexia nervosa. Am J Psychiatry 2017;174:850-8.

42. International Obsessive Compulsive Disorder Foundation Genetics C. Studies OCDCGA. Revealing the complex genetic architecture of obsessive-compulsive disorder using meta-analysis. Mol Psychiatry 2018;23:1181-8.

43. Zhan X, Hu Y, Li B, et al. RVTESTS: an efficient and comprehensive tool for rare variant association analysis using sequence data. Bioinformatics 2016;32:1423-6.

44. Yang L, Liu CC, Zheng H, et al. LRP1 modulates the microglial immune response via regulation of JNK and NF-kappaB signaling pathways. J Neuroinflammation 2016;13:304.

45. Mantuano E, Brifault C, Lam MS, et al. LDL receptor-related protein-1 regulates NFkappaB and microRNA-155 in macrophages to control the inflammatory response. Proc Natl Acad Sci U S A 2016;113:1369-74.

46. Chuang TY, Guo Y, Seki SM, et al. LRP1 expression in microglia is protective during CNS autoimmunity. Acta Neuropathol Commun 2016;4:68.

47. Purcell SM, Moran JL, Fromer M, et al. A polygenic burden of rare disruptive mutations in schizophrenia. Nature 2014;506:185-90.

48. Ganna A, Genovese G, Howrigan DP, et al. Ultra-rare disruptive and damaging mutations influence educational attainment in the general population. Nat Neurosci 2016;19:1563-5.

49. Donovan AP, Basson MA. The neuroanatomy of autism - a developmental perspective. J Anat 2017;230:4-15.

50. Auderset L, Cullen CL, Young KM. Low density lipoprotein receptor related protein 1 is differentially expressed by neuronal and glial populations in the developing and mature mouse central nervous system. PLoS One 2016;11:e0155878.

51. Zlokovic BV, Deane R, Sagare AP, et al. Low-density lipoprotein receptor-related protein-1: a serial clearance homeostatic mecha- nism controlling Alzheimer's amyloid beta-peptide elimination from the brain. J Neurochem 2010;115:1077-89.

52. Jeon H, Meng W, Takagi J, et al. Implications for familial hypercholesterolemia from the structure of the LDL receptor YWTD-EGF domain pair. Nat Struct Biol 2001;8:499-504.

53. Yamanaka Y, Faghihi MA, Magistri M, et al. Antisense RNA controls LRP1 sense transcript expression through interaction with a chromatin-associated protein, HMGB2. Cell Reports 2015; 11:967-76.

54. Kolb M, Kurz S, Schafer A, et al. Verification and characterization of an alternative low density lipoprotein receptor-related protein 1 splice variant. PLoS One 2017;12:e0180354.

55. Gan M, Jiang P, McLean P, et al. Low-density lipoprotein receptorrelated protein 1 (LRP1) regulates the stability and function of GluA1 alpha-amino-3-hydroxy-5-methyl-4-isoxazole propionic acid (AMPA) receptor in neurons. PLoS One 2014;9:e113237.

56. Masi A, Glozier N, Dale R, et al. The immune system, cytokines, and biomarkers in autism spectrum disorder. Neurosci Bull 2017; 33:194-204.

57. Liu CC, Hu J, Tsai CW, et al. Neuronal LRP1 regulates glucose metabolism and insulin signaling in the brain. J Neurosci 2015;35:5851-9.

58. Liu Q, Trotter J, Zhang J, et al. Neuronal LRP1 knockout in adult mice leads to impaired brain lipid metabolism and progressive, age-dependent synapse loss and neurodegeneration. J Neurosci 2010;30:17068-78.

59. Ionita-Laza I, Makarov V, Consortium AAS, et al. Scan-statistic approach identifies clusters of rare disease variants in LRP2, a gene linked and associated with autism spectrum disorders, in three datasets. Am J Hum Genet 2012;90:1002-13.

60. Li M, Huang L, Grigoroiu-Serbanescu M, et al. Convergent lines of evidence support $\operatorname{lrp} 8$ as a susceptibility gene for psychosis. Mol Neurobiol 2016;53:6608-19.

\section{We believe in open access to research}

To ensure continued worldwide free access to all JPN content, Research and Review articles accepted for publication are subject to an article processing fee of $\$ 1950$, payable on acceptance.

\section{Benefits of open access}

- For researchers and institutions: increased visibility, usage and impact for their work

- For government: a better return on investment for funding research

- For society: efficient, effective patient care resulting in better outcomes

JPN articles are available free of charge on the journal website (jpn.ca) and in PubMed Central. 